\title{
BMJ Open Practicability of lower extremity functional performance tests and their measurement properties in elite athletes: protocol for a systematic review
}

\author{
Rosalyn Cooke (D) , ${ }^{1}$ Alison Rushton (D) , ${ }^{2}$ James Martin (D) , ${ }^{3}$ Lee Herrington (D) , \\ Nicola R Heneghan (D) ${ }^{1}$
}

To cite: Cooke R, Rushton A, Martin J, et al. Practicability of lower extremity functional performance tests and their measurement properties in elite athletes: protocol for a systematic review. BMJ Open 2020;10:e042975. doi:10.1136/ bmjopen-2020-042975

- Prepublication history for this paper is available online. To view these files, please visit the journal online (http://dx.doi org/10.1136/bmjopen-2020042975).

Received 20 July 2020 Revised 03 November 2020 Accepted 04 November 2020

Check for updates

(C) Author(s) (or their employer(s)) 2020. Re-use permitted under CC BY-NC. No commercial re-use. See rights and permissions. Published by BMJ.

For numbered affiliations see end of article.

Correspondence to

Rosalyn Cooke;

rcc004@student.bham.ac.uk

\section{ABSTRACT}

Introduction Lower extremity injury (LEl) is highly prevalent and its occurrence increases the risk of future injury in athletic populations. Identifying athletes at risk of injury is the key to target injury-prevention programmes. Functional performance tests (FPT) assess an athlete's ability to produce and accept forces during movement tasks reflective of those experienced in sport, and are used to identify deficits in physical qualities or neuromuscular control. This review aims to identify FPT which have potential to predict LEl and assess their measurement properties associated with reliability, validity, responsiveness and practicability (interpretability and feasibility).

Methods/analysis This protocol will be reported using the Preferred Reporting Items for Systematic Review and Meta-Analysis Protocol and the COnsensus-based Standards for the selection of health Measurement INstruments Methodology. The search strategy has two stages: stage 1 will identify lower limb FPT used in athletic populations; and stage 2 will assess the measurement properties of the identified FPT. A sensitive search strategy will use MEDLINE, EMBASE, CINHAL and SPORTdiscus databases; from inception to June 2020. Hand searching reference lists, key journals and grey literature will be completed. One reviewer will complete search 1 and data extraction. Two reviewers will complete the search, data extraction and risk-of-bias assessment for search 2. Evidence will be pooled or summarised by individual measurement property by each individual study and grouped by FPT. Meta-analysis using a random effects model with subgroup analysis will be performed where possible. Pooled or summarised results for each FPT in relation to each measurement property will be rated against the criteria for good measurement properties. Two reviewers will assess the overall body of evidence per measurement property per FPT using the modified Grading of Recommendations, Assessment, Development and Evaluation guidelines. This review will enable clinicians to make an informed choice when selecting FPT.

Ethics and dissemination № ethical approval is required for this review and the results will be disseminated through peer-reviewed publications and submitted for conference presentation.

PROSPERO registration number CRD42020188932.
Strengths and limitations of this study

- This review will, for the first time, identify, assess quality, and report the available evidence for functional performance tests (FPT) that have the potential to predict a lower extremity injury (LEI) in elite athletic populations.

- A two-staged process will enable a sensitive search to first identify FPTs and second to evaluate their measurement properties.

- The COnsensus-based Standards for the selection of health Measurement INstruments risk-of-bias tool and modified Grading of Recommendations, Assessment, Development and Evaluation approach will be used.

- Findings will enable practitioners to select tests which have the ability to predict LEl in an elite athletic population.

\section{INTRODUCTION}

Lower extremity injury (LEI) represents a significant burden in athletic populations across sports.

Reviews of injury surveillance data within the National Collegiate Athletic Association consistently demonstrate LEI to account for more than $50 \%$ of total injuries. ${ }^{1-5}$ The trend of high rates of LEI is also seen in other sporting cohorts. LEI accounted for $57 \%$ of injuries reported in the Union of European Football Associations (UEFA) Elite Club Injury study ${ }^{6}$ with thigh $(34 \%)$, knee $(12 \%)$ and hip/groin $(11 \%)$ among the most common during 2018/2019 men's season. Within women's football, LEIs are the most common injuries and often more severe. ${ }^{257-10}$ Female players experience greater time loss and longer return to play times, for thigh and calf muscle strains and anterior cruciate ligament (ACL) injuries compared with male players. ${ }^{10}$ Female athletes have significant differences in relation to their anatomy, 
hormonal profile and neuromuscular qualities compared with male athletes, which have been linked to high injury risk movement patterns. ${ }^{11-18}$ This may explain why across sports, female athletes present with more severe LEI to the knee and ankle and with greater time loss from training and competition. ${ }^{2-5}$ 9-13 19 Previous LEI has been identified as a risk for future injury which when it occurs, may be more severe, may require surgical intervention and a prolonged rehabilitation period. ${ }^{20-23}$ Prevention of a primary LEI may lead to a healthier athletic population who are able to complete greater volumes and intensity of training, which have been shown to decrease future injury rate and improve performance. ${ }^{24-27}$

Evaluation of physical status is assessed on commencement and continuously throughout athletic careers. Data are used to assess physical qualities, inform training prescription and exposure to training load..$^{26}{ }^{28}$ It also informs interventions and rehabilitation as part of return to training and performance. ${ }^{2-35}$ Traditional clinical assessment techniques such as joint range of motion, ligament laxity and manual muscle testing have limited value to determine an athlete's injury risk. ${ }^{16} 323637$ Laboratory testing is considered the 'gold standard' when analysing movement patterns and forces that are potentially injurious, but methods are often expensive and require laboratory time. ${ }^{38}{ }^{39}$ Field-based testing also permits larger numbers of athletes to be regularly profiled without the need for specialist skills or expensive and time intensive methods such as three-dimensional motion capture and kinematic analysis. ${ }^{39-44}$

Functional performance tests (FPT) ${ }^{3745}$ are quantitative measures used to define function and/or outcome. FPT include assessment of an individuals' ability to coordinate their neuromuscular system to produce, accept and adapt to multiplanar forces occurring in movement patterns which mimic or are similar to those required for sporting performance. There are a variety of terms to describe this form of assessment including field expedient tests, ${ }^{46}$ functional outcome measures and physical performance tests. ${ }^{47}{ }^{48}$ FPT such as Functional Hop Test, ${ }^{49}$ Landing Error Scoring System ${ }^{50}$ and Star Excursion Balance Test $^{5253}$ are commonly used to assess a specific condition (eg, chronic ankle instability ${ }^{52}$ 54, ACL injury ${ }^{355-59}$ ) or specific cohorts of athletes (eg, female basketball players, prepubescent athletes). ${ }^{45} 55-64$

Poor performance in FPT has been linked to increased risk of injury due to a deficit in physical qualities or neuromuscular control. The Y-balance test is associated with increased injury risk when there is a difference of $>4 \mathrm{~cm}$ between limbs. ${ }^{48} 52$ Combining FPT into a testing battery to assess a variety of physical and movement qualities has shown capacity to identify athletes at risk of LEI. ${ }^{45566}$ Brumitt et $a l^{65}$ tested three FPT in preseason with a cohort of 106 division III female athletes across 8 sports. They were able to identify risk profiles based on two or more suboptimal scores which were associated with an increased risk of LEI and time loss from training/ competition. Categorising athletes into high and low risk groups enables targeted injury-prevention interventions which in turn may be a more cost-effective use of healthcare resources through decreasing injury rates. ${ }^{456566}$

Knowledge of FPT measurement properties is essential to evaluate the quality of the results produced by the FPT and if it is appropriate for its intended use. This enables understanding of the influence of individual FPT and how they contribute to a composite score such as the Functional Movement Score. ${ }^{4565-69}$ The COnsensusbased Standards for the selection of health Measurement INstruments (COSMIN) taxonomy of measurement properties has three domains which are reliability, validity and responsiveness. ${ }^{70}$ Further subgroupings of interpretability and feasibility are also described within the COSMIN systematic review methodology. ${ }^{71}$ Interpretability and feasibility can be combined to form the domain practicability which is important when considering which FPT to use in the field. ${ }^{39}$ COSMIN methodology applies a robust and rigorous assessment of measurement properties and a systematic review using this approach is required to inform practitioners and researchers which FPT can be used with athletic populations to identify those at risk of LEI.

\section{AIM}

To identify, evaluate and report FPT that identify athletes at risk of, or who sustain an LEI, and report their measurement properties.

Objectives

1. To identify FPT that have been used in elite athletic population to identify individuals at risk of sustaining an LEI.

2. To evaluate and report for each FPT: reliability (internal consistency, test-retest, inter-rater and intrarater), validity (content, structural or criterion), measurement error, responsiveness and practicability (interpretability/feasibility)

3. To synthesise the available evidence, assess and report the quality of FPT measurement properties using the modified Grading of Recommendations, Assessment, Development and Evaluation (GRADE) criteria.

\section{METHODS}

\section{Design, protocol and registration}

This systematic review protocol has been designed using the Preferred Reporting Items for Systematic review and Meta-Analysis Protocol (PRISMA-P) ${ }^{72}$ and COSMIN methodology for a systematic review of outcome measures. ${ }^{71}$ The protocol is registered on PROSPERO and is reported in line with PRISMA-P. The systematic review will be conducted in two stages with two searches. Search 1 will identify the FPT used to predict LEI (objective 1), and search 2 will use the identified FPT as search terms to identify studies which evaluate measurement properties of the measures (objective 2). Modified GRADE criteria 
Table 1 Inclusion/exclusion criteria for search 1: Identification of FPT

\begin{tabular}{|c|c|c|}
\hline Components & Inclusion criteria & Exclusion criteria \\
\hline Construct & $\begin{array}{l}\text { The FPT have predicted musculoskeletal injury to lower } \\
\text { extremity - acute, chronic or recurrent } \\
\text { The FPT have ability to differentiated between injured and } \\
\text { non-injured athletes }\end{array}$ & - Prediction of secondary injury \\
\hline Population & $\begin{array}{l}\text { Athletes (elite, pre-elite, collegiate) } \\
>16 \text { years old }\end{array}$ & $\begin{array}{l}\text { Non-athletic populations in } \\
\text { rehabilitation settings } \\
\text { - Recreational level athletes }\end{array}$ \\
\hline Study design & $\begin{array}{l}\text { Randomised control trial } \\
\text { Cohort studies } \\
\text { Observational studies } \\
\text { Prospective study design }\end{array}$ & - Case studies \\
\hline
\end{tabular}

FPT, functional performance tests.

will then be applied to the evidence synthesised from search 2 (objective 3 ).

\section{Eligibility criteria}

\section{Search 1: identification of FPT}

Search 1 will identify relevant FPT which are predictive of LEI in an athletic population. Table 1 outlines the eligibility criteria for article selection. These criteria were informed by the key elements outline in the COSMIN methodology for systematic reviews. ${ }^{71}$

\section{Search 2: measurement properties of FPT}

Following search 1, the identified FPT will be searched for within the literature using search terms in relation to FPT naming conventions, lower limb injury and measurement properties. Measurement properties of the FPT that have demonstrated ability to predict injury are the outcomes of interest as outlined in table 2. These criteria were informed by the key elements outline in the COSMIN methodology for systematic reviews. ${ }^{71} 72$

\section{Patient and public involvement}

The study was developed as a result of conversations with athletes and coaching staff about the value of the information generated through screening for injury risk. The athlete's perspective is that they want to know how they have performed in relation to their teammates, peers and competitors. While coaching staff wish to know that the time invested in screening is providing information that is useful and informs training and performance. These two different perspectives have informed the question and design of the review in an attempt to answer this. No athlete data will be required during data collection or analysis but the results of this review will be made available for athletes, coaches and support staff in sports where LEIs are a significant burden.

\section{Information sources}

The following databases will be used in the search: MEDLINE, EMBASE, Cumulative Index to Nursing and Allied Health Literature, and SPORTDiscus. These databases will be used for both stages of searches. The databases outlined in the search strategy will be used in both searches 1 and 2. Hand searching will include the reference lists from articles identified in both searches. Hand searching for the timespan of 1991-2020 of the following journals will be completed: British Journal of Sports and exercise Medicine, Physical Therapy in Sport, Journal of Strength and Conditioning research, Journal of Orthopaedic and Sports Physical therapy and Journal of Athletic Training. Grey Literature will be searched using the British national bibliography for report literature, electronic thesis online service for dissertations and abstracts and Open Grey.

\section{Search strategy}

In order to identify relevant studies and to achieve a comprehensive systematic review, a reproducible search strategy has been devised and completed by one reviewer (RC) for search 1 and two reviewers (RC and LH) for search 2. The search strategy will use MEDLINE terms and keywords and the search strategy is detailed in box 1 . Search terms will be amended for individual differences of each database as required. ${ }^{71}$ For search 2, a search filter for measurement properties will be applied in appropriate databases to improve the accuracy. ${ }^{73}$ For both searches there will be no limitations to date of publication; however, only human studies and those reported in English, and where there is access to full-text articles, will be included and reviewed. 


\begin{tabular}{|c|c|c|}
\hline Components & Inclusion criteria & $\begin{array}{l}\text { Exclusion } \\
\text { criteria }\end{array}$ \\
\hline Construct & $\begin{array}{l}\text { Reports } \\
\text { measurement } \\
\text { properties related } \\
\text { to } \\
\text { Reliability } \\
\text { - Validity } \\
\text { Responsiveness } \\
\text { - Practicability } \\
\text { (Interpretability/ } \\
\text { feasibility) }\end{array}$ & $\begin{array}{l}\text { Solely } \\
\text { describes the } \\
\text { test }\end{array}$ \\
\hline Population & $\begin{array}{l}\text { Athletes (elite, } \\
\text { pre-elite, } \\
\text { collegiate) } \\
>16 \text { years old }\end{array}$ & $\begin{aligned} & \text { Non-athletic } \\
& \text { populations in } \\
& \text { rehabilitation } \\
& \text { settings } \\
& \text { Recreational } \\
& \text { level athletes }\end{aligned}$ \\
\hline Types of instrument & $\begin{array}{l}\text { FPT of the } \\
\text { lower extremity } \\
\text { (hip terminal } \\
\text { phalanges of } \\
\text { foot) } \\
\text { FPT which } \\
\text { can feasibly } \\
\text { be completed } \\
\text { in the field of } \\
\text { play requiring } \\
\text { minimal } \\
\text { equipment }\end{array}$ & \\
\hline Study design & $\begin{array}{l}\text { Randomised } \\
\text { control trial } \\
\text { Cohort studies } \\
\text { Observational } \\
\text { studies } \\
\text { Prospective } \\
\text { study design }\end{array}$ & - Case studies \\
\hline Limits & $\begin{array}{l}\text { Studies reported } \\
\text { in English }\end{array}$ & \\
\hline
\end{tabular}

FPT, functional performance tests.

\section{Study records}

Data management

Results from searches will be imported into End Note V.X8 (Clarivate Analytics) reference management software.

\section{Selection process}

A PRISMA flow chart (figure 1) presents the number of papers identified by both searches and those which were included in the final review with reasons for exclusion detailed.

Titles and abstracts will be screened by two reviewers ( $\mathrm{RC}$ and LH) independently for inclusion. Where a study achieves 'undecided' at this first review a full text will be requested in order for a decision to be made. Full text of

\section{Box 1 Search strategy for MEDLINE}

\section{Search 1}

1. lower extremity.mp.

2. exp lower extremity/

3. exp lower limb/

4. hip.mp

5. knee.mp

6. ankle.mp

7. foot.mp

8. exp anterior cruciate ligament

9. $\exp$ acl

10. exp lateral ankle ligament

11. exp chronic ankle

12. injury.mp.

13. exp injury/

14. ligament injuries.mp

15. joint instability.mp

16. strain/sprain.mp

17. exp strain/sprain

18. Athlete.mpathlete.mp.

19. exp Athleteathlete/

20. screening.mp.

21. exp screening/

22. preparticipation screening.mp

23. physical performance test.mp.

24. functional performance test.mp.

25. functional outcome measure.mp

26. field expedient test.mp

27. movement screen.mp.

28. functional movement score.mp

29. exp hop test/

30. exp fitness test/

31. exp single leg squat

32. exp vertical jump

33. exp star excursion balance test

34. $\exp Y$ balance test

35. exp tuck jump

36. exp landing error scoring system

37. $1-7 / 0 R$

38. $8-17 / 0 R$

39. 18 OR 19

40. $20-34 / 0 R$

41. 35 AND 36 AND 37 and 38

42. Limit 39 to Humans

43. Limit 40 to $>16$ years old

Search 2

1. Name of Identified functional performance test (example: $Y$ balance test)

2. Lower extremity.mp.

3. exp lower extremity/

4. exp lower limb/

5. hip.mp

6. knee.mp

7. ankle.mp

8. foot.mp

9. exp anterior cruciate ligament

10. $\exp$ acl

11. exp lateral ankle ligament

12. exp chronic ankle

13. injury.mp. 


\section{Box 1 Continued}

\section{4. exp injury/}

15. ligament injuries.mp

16. joint instability.mp

17. strain/sprain.mp

18. exp strain/sprain

19. Athlete.mp.

20. exp Athlete/

21. validity.mp

22. exp validation studies/

23. reliability.mp

24. exp reproducibility of results/

25. interpretability.mp

26. internal consistency.mp

27. exp sensitivity and Specificity/

28. clinical sensitivity.mp

29. exp psychometrics/

30. responsiveness.mp

31. exp Evaluation studies/

32. measurement error.mp

33. measurement properties.mp

34. $42-53 / 0 R$

35. $54-59 / 0 R$

36. $60 \mathrm{OR} 61$

37. $62-74 / 0 R$

38. 75 AND 76 AND 77 AND 78

39. Limit 79 Humans

40. Limit 80 to $>16$ years old

all potential eligible studies will then be screened independently (RC \& LH) with a third reviewer (NRH) acting as a mediator in the event of disagreement. Agreement by reviewers at first and second review will be assessed by kappa statistic using previously reported levels of 0.40 0.59 fair, $0.60-0.74$ good and 0.75 or more as excellent agreement. $^{74}$

\section{Data collection process}

Data will be extracted independently by one reviewer for search 1 (RC) and two reviewers (RC and LH) from the articles identified in search 2 . The data extraction form will be trialled on three randomly selected papers from search 2 to ensure that all relevant information is collected. Contact with authors will be made via email if required. This will be to seek clarification on FPT methodology or for raw data to enable further analysis such as calculation of measurement error. ${ }^{74}$

\section{Data items}

Table 3 summarises the data items that will be extracted from the full-text articles.

\section{Risk of bias in individual studies}

The COSMIN risk-of-bias checklist ${ }^{75}$ will be used to assess methodological quality of measurement properties within individual studies. Two reviewers (RC and LH) will independently assess included studies from searches 1 and 2. In the event of disagreement, a third reviewer (NRH) will mediate the decision. For each study, the checklist will be used to assess the methodological quality for each measurement property separately. Each measurement property will be rated as very good, adequate, doubtful or inadequate quality. ${ }^{716}$ The overall methodological quality of individual studies for each measurement property will be rated based on 'the worst score counts principle'. ${ }^{71}$ The ratings for each measurement property per individual study, as well as the overall rating of the individual study, will be presented in the final results. ${ }^{71}$

\section{Data synthesis}

In line with COSMIN guidelines for systematic reviews, ${ }^{71}$ the results will be quantitatively pooled or qualitatively summarised for each measurement property, and grouped per each FPT. FPT protocols may demonstrate variation between studies, and therefore FPT will be grouped by movement task. An overall rating of these pooled or summarised results will be assessed using the updated criteria for good measurement properties ${ }^{76}$ as sufficient $(+)$, insufficient $(-)$ or indeterminate (?). The quality of evidence will be assessed using the modified GRADE approach. ${ }^{71}$ Summary of findings tables will be used to present these results. Where possible a random effects meta-analysis will be conducted on the pooled estimates of different studies (minimum of 2) for each measurement property using the DerSimonian-Laird method. ${ }^{77}$ In the presence of high heterogeneity where $\mathrm{I}^{2}>75 \%,{ }^{78}$ further analysis will be considered using the following subgroups, (1) specific athlete cohort, for example, female, (2) injury diagnosis for example, ACL, (3) lower extremity anatomical location (foot, ankle, knee and hip) and (4) sport.

If quantitative pooling is not possible due to limited numbers of studies or high heterogeneity, qualitative summarising of the results will be completed and report information such as range of scores for minimally important change (MIC) to assess interpretability. ${ }^{71}$ For the summarised results to be considered sufficient, at least $75 \%$ of the results must meet the criteria for good measurement properties. ${ }^{76}$ The overall rating of the pooled or summarised result will be added to the summary of findings table per measurement property per FPT.

For an FPT to be recommended for use in profiling athletes, it must demonstrate sufficient content validity and at least low-quality evidence for sufficient internal consistency. ${ }^{71}$

Practicability of the FPT is an essential criterion as well as the quality of the measurement properties associated with it. For this review practicability will be assessed by two reviewers with extensive experience of completing FPT with elite athletes and will include factors relating to the interpretability (complexity of FPT scoring, minimal detectable change and MIC) and feasibility (test duration, equipment required, cost, scoring and completion complexity). ${ }^{39} 79$ 
Search 1

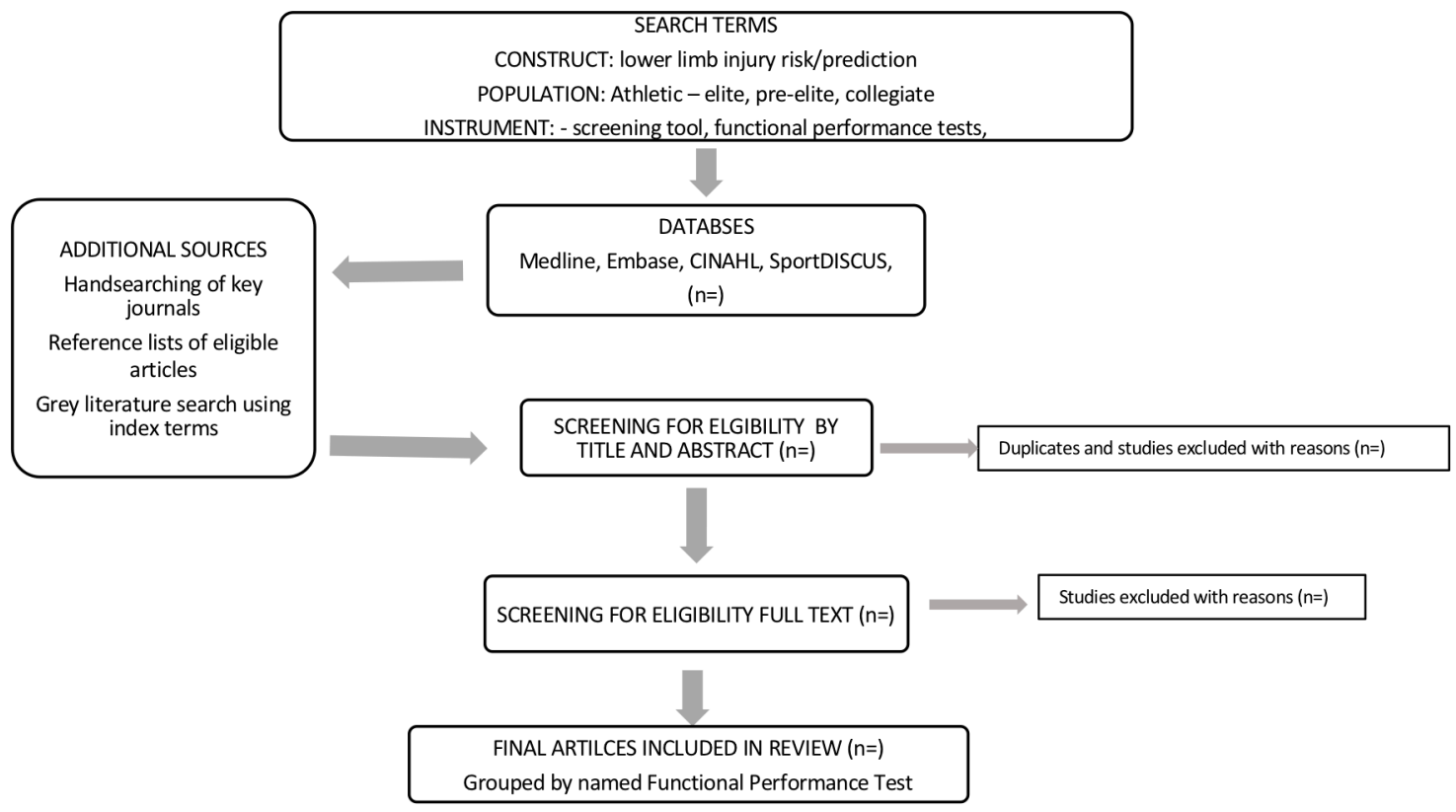

Search 2

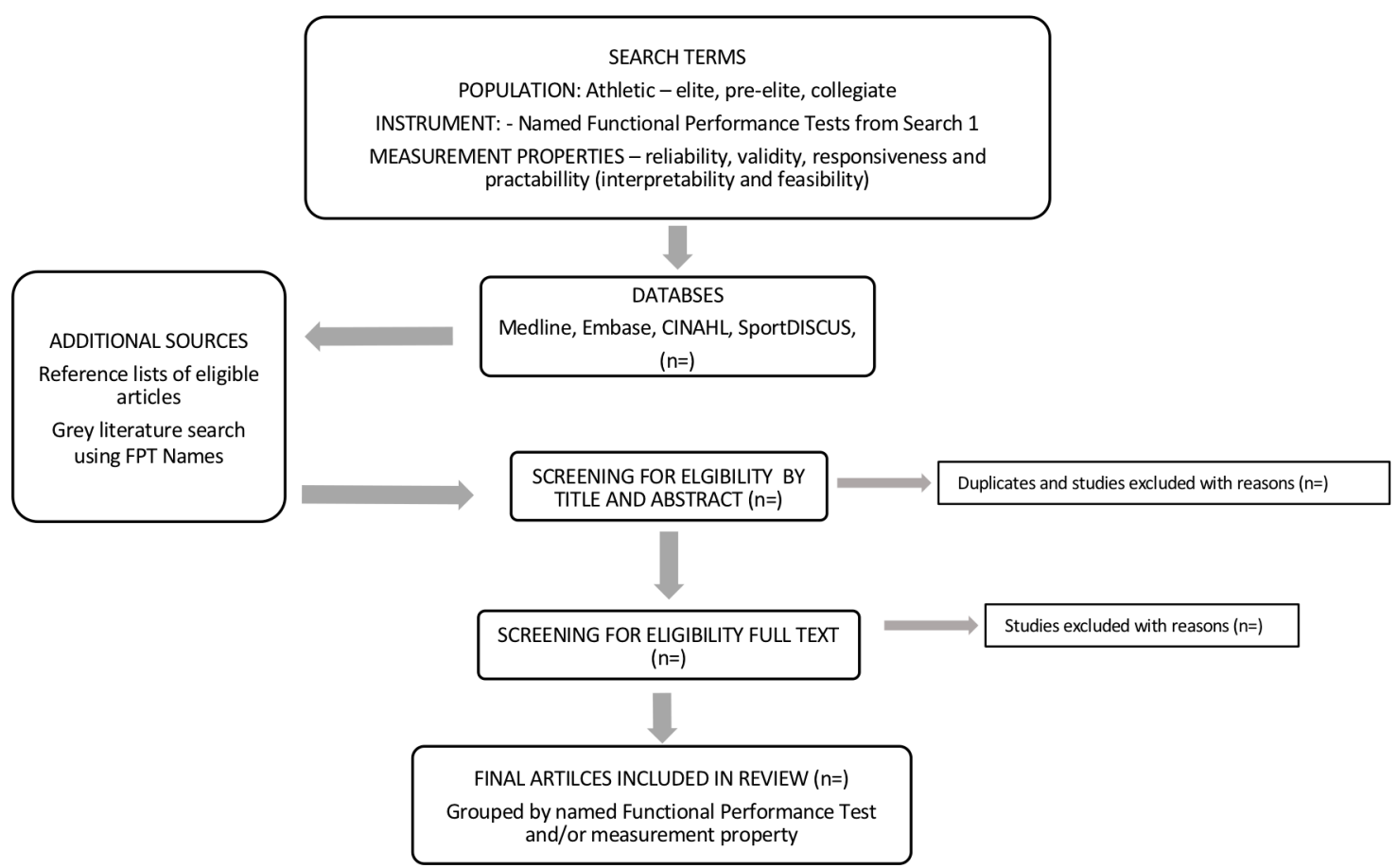

Figure 1 Flow chart of search strategy and study selection process.

\section{Confidence in cumulative evidence}

A modified GRADE approach ${ }^{71}$ will be used to assess the overall body of evidence for the measurement properties of the identified FPT. This approach differs from the original GRADE criteria and only uses the following factors as it basis of assessment: (1) risk of bias (quality of studies), (2)inconsistency (of the results of the studies), (3) indirectness of evidence (differences in population, 
Table 3 Data items for extraction: search 1 and search 2

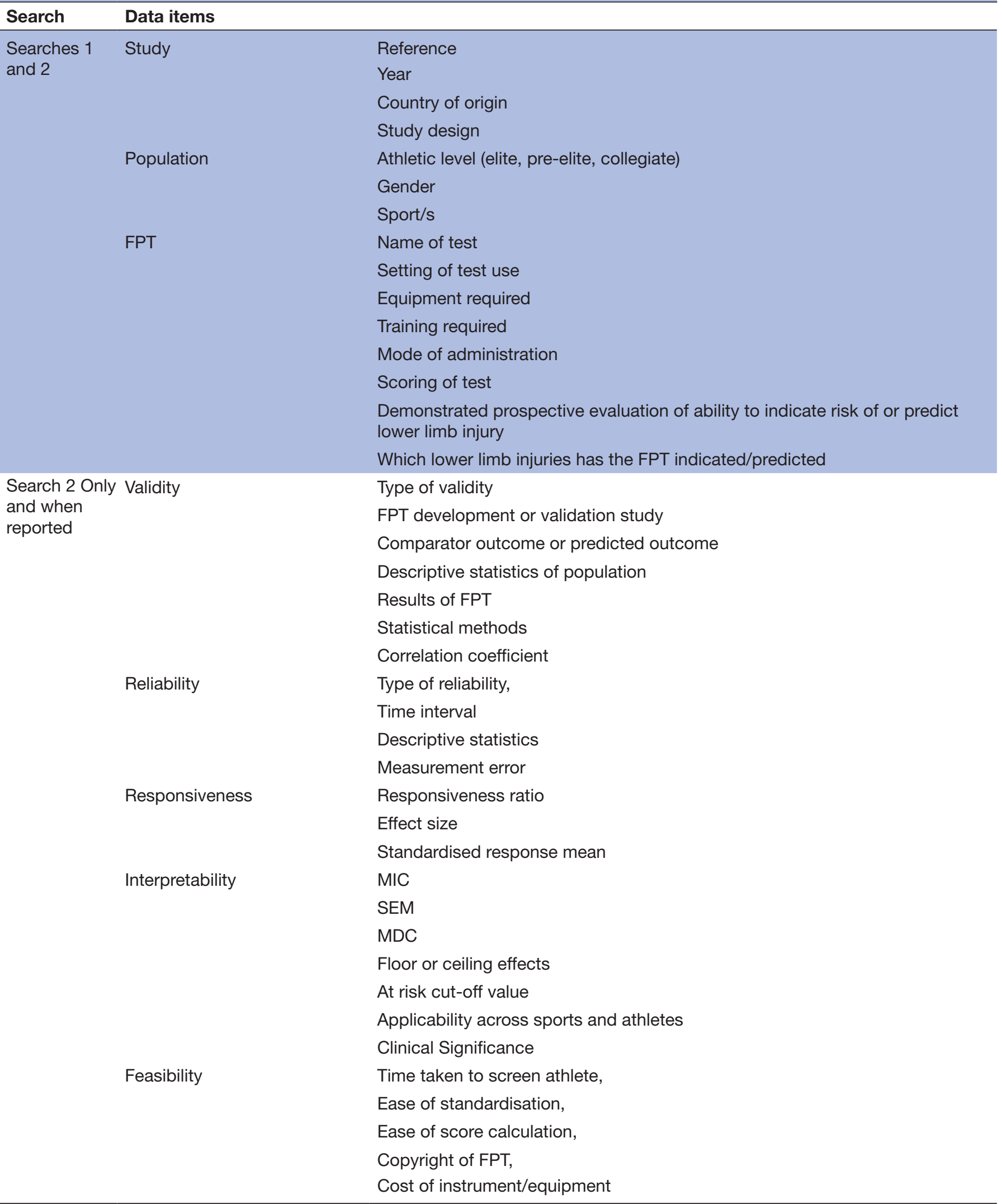

FPT, functional performance tests; MDC, minimal detectable change; MIC, minimally important change; SEM, SE of measurement. 
construct than ones of interest in the review) and (4) imprecision of effect estimates (wide CIs). The fifth factor used in the GRADE approach assesses publication bias which is not included by the COSMIN methodology due to the lack of registry for measurement properties. ${ }^{71}$ This process will be completed by two reviewers (RC and LH) with the support of a third reviewer (NRH) to achieve consensus if required.

\section{DISCUSSION}

FPT assess an individual's ability to perform movement patterns and adapt to multiplanar forces that are similar to those experienced during sporting activity. ${ }^{37} 45-53$ The ability to assess this outside of the biomechanics laboratory provides practitioners and researchers with low tech, low cost options to profile large numbers of athletes at regular time intervals. ${ }^{40-45}$ Being able to identify those athletes at risk enables targeted injury-prevention programmes to be implemented. 303945616567

FPT for the lower extremity have been used in a variety of contexts in both non-injured and rehabilitation populations with different methodologies. In light of this, there is a need for a specific review to identify which FPT identify athletes at risk of LEI, assess the measurement properties of these FPT, and report their quality and practicability.

This review through its comprehensive and robust methods will seek to synthesise the available evidence for FPT in this context. The anticipated outputs from this review will recommend FPT which have the ability to identify individuals at risk of sustaining an LEI. As this review considers a specific population and using FPT to prospectively predict injury the number, variability in FPT test protocol and methodological quality of the available studies may limit the potential for meta-analysis. To overcome this limitation a comprehensive narrative synthesis informed by the available results on measurement properties will be completed and priorities for future research will be reported.

\section{Implications of study}

Previous LEI predisposes athletes to future injury which results in further time loss from training and competition compromising their sporting potential or even leading to their retirement from sport. ${ }^{20-23}$ Identifying those athletes at risk of sustaining an LEI is a priority and FPT offer the ability to profile athletes without the requirements for high cost, time intensive laboratory-based testing. ${ }^{40-45}$

A systematic review is needed to evaluate which FPT have the ability to identify athletes at risk of LEI. The results of this review will enable practitioners and researchers to select the best available FPT based on their measurement properties and practicability. The results of this review may also highlight the need to optimise or standardise FPT testing protocols and results reporting to address deficits in measurement properties as well as enable a comparison between different athletic cohorts.

\section{Amendments}

Any changes to this protocol will be reported in the final review as well as detailed on PROSPERO. Information will include the date, the changes and the rationale for these.

\section{ETHICS AND DISSEMINATION}

No ethics approval is required for this systematic review. The results of this systematic review will be disseminated through peer-reviewed journals as well as international and national conferences presentation.

\section{Author affiliations}

${ }^{1}$ Centre of Precision Rehabilitation for Spinal Pain (CPR Spine), School of Sport, Exercise and Rehabilitation Sciences, College of Life and Environmental Sciences, University of Birmingham, Birmingham, UK

${ }^{2}$ School of Physical Therapy, Faculty of Health Sciences, Western University, London, Ontario, Canada

${ }^{3}$ Institute of Applied Health Research, Public Health Building, College of Medical and Dental Sciences, University of Birmingham, Birmingham, UK

${ }^{4}$ School of Health and Society, University of Salford, Salford, UK

Twitter Rosalyn Cooke @RosCooke1 and Nicola R Heneghan @HeneghanNicola

Contributors All authors conceptualised and designed the protocol. $\mathrm{RC}$ is a $\mathrm{PhD}$ student and NRH (lead supervisor), AR, JM and LH are supervisors. RC drafted the initial manuscript with NRH, AR, JM and LH providing guidance on design, topic, methodology and analyses. All authors reviewed and commented on each draft of the protocol. All authors have approved and contributed to the final manuscript.

Funding The authors have not declared a specific grant for this research from any funding agency in the public, commercial or not-for-profit sectors.

Competing interests None declared.

Patient and public involvement Patients and/or the public were involved in the design, or conduct, or reporting, or dissemination plans of this research. Refer to the Methods section for further details.

Patient consent for publication Not required.

Provenance and peer review Not commissioned; externally peer reviewed.

Open access This is an open access article distributed in accordance with the Creative Commons Attribution Non Commercial (CC BY-NC 4.0) license, which permits others to distribute, remix, adapt, build upon this work non-commercially, and license their derivative works on different terms, provided the original work is properly cited, appropriate credit is given, any changes made indicated, and the use is non-commercial. See: http://creativecommons.org/licenses/by-nc/4.0/.

\section{ORCID iDs}

Rosalyn Cooke http://orcid.org/0000-0003-2784-9365 Alison Rushton http://orcid.org/0000-0001-8114-7669 James Martin http://orcid.org/0000-0002-6949-4200

Lee Herrington http://orcid.org/0000-0003-4732-1955

Nicola R Heneghan http://orcid.org/0000-0001-7599-3674

\section{REFERENCES}

1 Hootman JM, Dick R, Agel J. Epidemiology of collegiate injuries for 15 sports: summary and recommendations for injury prevention initiatives. J Ath/ Train 2007;42:311-9.

2 DiStefano LJ, Dann CL, Chang CJ, et al. The first decade of webbased sports injury surveillance: descriptive epidemiology of injuries in US high school girls' soccer (2005-2006 through 2013-2014) and national collegiate athletic association women's soccer (2004-2005 through 2013-2014). J Athl Train 2018;53:880-92.

3 Clifton DR, Hertel J, Onate JA, et al. The first decade of web-based sports injury surveillance: descriptive epidemiology of injuries in US high school girls' Basketball (2005-2006 through 2013-2014) and national collegiate athletic association women's Basketball (20042005 through 2013-2014). J Athl Train 2018;53:1037-48.

4 Lynall RC, Gardner EC, Paolucci J, et al. The first decade of web-based sports injury surveillance: descriptive epidemiology 
of injuries in US high school girls' field hockey (2008-2009 through 2013-2014) and national collegiate athletic association women's field hockey (2004-2005 through 2013-2014). J Athl Train 2018:53:938-49.

5 Roos KG, Wasserman EB, Dalton SL, et al. Epidemiology of 3825 injuries sustained in six seasons of National Collegiate Athletic Association men's and women's soccer (2009/2010-2014/2015). Br J Sports Med 2017;51:1029-34.

6 UEFA elite Club injury study: 2018/19 season report. 2019 Jun 28;:147. Available: https://www.uefa.com/MultimediaFiles/Download/ uefaorg/Medical/02/61/67/86/2616786_DOWNLOAD.pdf [Accessed March 2020].

7 Prien A, Prinz B, Dvořák J, et al. Health problems in former elite female football players: prevalence and risk factors. Scand J Med Sci Sports 2017;27:1404-10.

8 Martínez-Lagunas V, Niessen M, Hartmann U. Women's football: Player characteristics and demands of the game. J Sport Health Sci 2014;3:258-72.

9 Montalvo AM, Schneider DK, Yut L, et al. "What's my risk of sustaining an ACL injury while playing sports?" A systematic review with meta-analysis. Br J Sports Med 2019;53:1003-12.

10 Larruskain J, Lekue JA, Diaz N, et al. A comparison of injuries in elite male and female football players: a five-season prospective study. Scand J Med Sci Sports 2018;28:237-45.

11 Joseph C, Naughton G, Antcliff A. Australian netball injuries in 2016: an overview of insurance data. Journal of Science and Medicine in Sport 2019;22:1304-8.

12 Reid DA, Vanweerd RJ, Larmer PJ, et al. The inter and intra rater reliability of the Netball movement screening tool. J Sci Med Sport 2015;18:353-7.

13 Attenborough AS, Sinclair PJ, Sharp T, et al. A snapshot of chronic ankle instability in a cohort of netball players. J Sci Med Sport 2016:19:379-83.

14 Alentorn-Geli E, Myer GD, Silvers HJ, et al. Prevention of noncontact anterior cruciate ligament injuries in soccer players. Part 1: mechanisms of injury and underlying risk factors. Knee Surg Sports Traumatol Arthrosc 2009;17:705-29.

15 Myer GD, Ford KR, Brent JL, et al. An integrated approach to change the outcome Part I. J Strength Cond Res 2012;26:2265-71.

16 Myer GD, Khoury J, Succop P, et al. Clinic-Based algorithm to identify female athletes at risk for anterior cruciate ligament injury: letter to the editor. Am J Sports Med 2013;41:NP1-6.

17 Hewett TE, Lynch TR, Myer GD, et al. Multiple risk factors related to familial predisposition to anterior cruciate ligament injury: fraternal twin sisters with anterior cruciate ligament ruptures. Br J Sports Med 2010;44:848-55.

18 Shultz SJ, Schmitz RJ, Benjaminse A, et al. Acl research retreat $\mathrm{Vl}$ : an update on ACL injury risk and prevention. J Athl Train 2012;47:591-603.

19 Smyth EA, Piromalli L, Antcliff A, et al. A prospective study of health problems at the $201817 / \mathrm{U}$ and 19/U Australian National Netball Championships with comparison of surveillance methodology. J Sci Med Sport 2020;23:215-21.

20 Hagglund M. Previous injury as a risk factor for injury in elite football: a prospective study over two consecutive seasons. Br J Sports Med 2006;40:767-72.

21 Hägglund $M$, Waldén M, Ekstrand J. Risk factors for lower extremity muscle injury in professional soccer. Am J Sports Med 2013:41:327-35.

22 Toohey LA, Drew MK, Cook JL, et al. Is subsequent lower limb injury associated with previous injury? A systematic review and metaanalysis. Br J Sports Med 2017:51:1670-8.

23 Hewett TE, Di Stasi SL, Myer GD. Current concepts for injury prevention in athletes after anterior cruciate ligament reconstruction. Am J Sports Med 2013:41:216-24.

24 Hägglund $M$, Waldén $M$, Magnusson $\mathrm{H}$, et al. Injuries affect team performance negatively in professional football: an 11-year followup of the UEFA champions League injury study. Br J Sports Med 2013;47:738-42.

25 Smyth EA, Newman P, Waddington G, et al. Injury prevention strategies specific to pre-elite athletes competing in Olympic and professional sports - a systematic review. J Sci Med Sport 2019;22:887-901.

26 Gabbett TJ, Nielsen RO, Bertelsen ML, et al. In pursuit of the 'Unbreakable' Athlete: what is the role of moderating factors and circular causation? Br J Sports Med 2019;53:394-5.

27 Gabbett T. Injury prevention and performance enhancement in team sports. Aspetar Sports Medicine Journal 2012;3:218-23.

28 Malone S, Hughes B, Doran DA, et al. Can the workload-injury relationship be moderated by improved strength, speed and repeated-sprint qualities? J Sci Med Sport 2019;22:29-34.
29 Creighton DW, Shrier I, Shultz R, et al. Return-to-play in sport: a decision-based model. Clin J Sport Med 2010;20:379-85.

30 Tassignon B, Verschueren J, Delahunt E, et al. Criteria-Based return to sport decision-making following lateral ankle sprain injury: a systematic review and narrative synthesis. Sports Med 2019;49:601-19.

31 Myer GD, Paterno MV, Ford KR, et al. Neuromuscular training techniques to target deficits before return to sport after anterior cruciate ligament reconstruction. $J$ Strength Cond Res 2008;22:987-1014.

32 Ardern CL, Glasgow P, Schneiders A, et al. 2016 consensus statement on return to sport from the first world Congress in sports physical therapy, Bern. Br J Sports Med 2016;50:853-64.

33 White K, Di Stasi SL, Smith AH, et al. Anterior cruciate ligamentspecialized post-operative return-to-sports (ACL-SPORTS) training: a randomized control trial. BMC Musculoskelet Disord 2013;14:108.

34 Barber-Westin SD, Noyes FR. Factors used to determine return to unrestricted sports activities after anterior cruciate ligament reconstruction. Arthroscopy 2011;27:1697-705.

35 Barber-Westin SD, Noyes FR. Objective criteria for return to athletics after anterior cruciate ligament reconstruction and subsequent Reinjury rates: a systematic review. Phys Sportsmed 2011;39:100-10.

36 Myer GD, Schmitt LC, Brent JL, et al. Utilization of modified NFL combine testing to identify functional deficits in athletes following ACL reconstruction. J Orthop Sports Phys Ther 2011;41:377-87.

37 Clark NC, Gumbrell CJ, Rana S, et al. Intratester reliability and measurement error of the adapted crossover hop for distance. Physical Therapy in Sport 2002;3:143-51.

38 Nilstad A, Andersen TE, Bahr R, et al. Risk factors for lower extremity injuries in elite female football players. $\mathrm{Br} \mathrm{J}$ Sports $\mathrm{Med}$ 2014;48:645.1-645.

39 Fox AS, Bonacci J, McLean SG, et al. A systematic evaluation of Field-Based screening methods for the assessment of anterior cruciate ligament (ACL) injury risk. Sports Med 2016;46:715-35.

40 Steffen K, Kroken T, Krosshaug T. Comparisons of 3D- and 2DMEASUREMENT techniques and observational real-time assessment of frontal plane knee control in a vertical drop jump landing. $\mathrm{Br} \mathrm{J}$ Sports Med 2014;48:662.3-3.

41 Weir G, Alderson J, Smailes N, et al. A reliable video-based ACL injury screening tool for female team sport athletes. Int J Sports Med 2019;40:191-9.

42 Munro A, Herrington L, Carolan M. Reliability of 2-Dimensional video assessment of Frontal-Plane dynamic knee valgus during common athletic screening tasks. J Sport Rehabil 2012;21:7-11.

43 McLean SG. Evaluation of a two dimensional analysis method as a screening and evaluation tool for anterior cruciate ligament injury. $\mathrm{Br}$ $J$ Sports Med 2005;39:355-62.

44 Munro A, Herrington L, Comfort P. The relationship between 2-Dimensional Knee-Valgus angles during Single-Leg squat Single-Leg-Land, and Drop-Jump screening tests. J Sport Rehabil 2017:26:72-7.

45 Clark N. Functional performance testing following knee ligament injury physical therapy in sport. Physical Therapy in Sport 2001;2:91-105

46 Lehr ME, Plisky PJ, Butler RJ, et al. Field-expedient screening and injury risk algorithm categories as predictors of noncontact lower extremity injury. Scand J Med Sci Sports 2013;23:e225-32.

47 Hegedus EJ, McDonough S, Bleakley C, et al. Clinician-friendly lower extremity physical performance measures in athletes: a systematic review of measurement properties and correlation with injury, part 1. The tests for knee function including the hop tests. Br J Sports Med 2015;49:642-8.

48 Hegedus EJ, McDonough SM, Bleakley C, et al. Clinician-friendly lower extremity physical performance tests in athletes: a systematic review of measurement properties and correlation with injury. Part 2-the tests for the hip, thigh, foot and ankle including the star excursion balance test. Br J Sports Med 2015;49:649-56.

49 Noyes FR, Barber SD, Mangine RE. Abnormal lower limb symmetry determined by function hop tests after anterior cruciate ligament rupture. Am J Sports Med 1991;19:513-8.

50 Padua DA, Marshall SW, Boling MC, et al. The landing error scoring system (less) is a valid and reliable clinical assessment tool of JumpLanding biomechanics. Am J Sports Med 2009;37:1996-2002.

51 Padua DA, Boling MC, Distefano LJ, et al. Reliability of the landing error scoring system-real time, a clinical assessment tool of jumplanding biomechanics. J Sport Rehabil 2011;20:145-56.

52 Gribble PA, Hertel J, Plisky P. Using the StAR excursion balance test to assess dynamic Postural-Control deficits and outcomes in lower extremity injury: a literature and systematic review. J Athl Train 2012;47:339-57. 
53 McGuine TA, Greene JJ, Best T, et al. Balance as a predictor of ankle injuries in high school basketball players. Clin J Sport Med 2000;10:239-44.

54 Delahunt E, Bleakley CM, Bossard DS, et al. Clinical assessment of acute lateral ankle sprain injuries (roast): 2019 consensus statement and recommendations of the International ankle Consortium. $\mathrm{Br} \mathrm{J}$ Sports Med 2018:52:1304-10.

55 Onate JA, Starkel C, Clifton DR, et al. Normative functional performance values in high school athletes: the functional PreParticipation evaluation project. J Athl Train 2018;53:35-42.

56 Hopper DM, Goh SC, Wentworth LA, et al. Test-retest reliability of knee rating scales and functional hop tests one year following anterior cruciate ligament reconstruction. Physical Therapy in Sport 2002;3:10-18.

57 Reid A, Birmingham TB, Stratford PW, et al. Hop testing provides a reliable and valid outcome measure during rehabilitation after anterior cruciate ligament reconstruction. Phys Ther 2007;87:337-49.

58 Gustavsson A, Neeter C, Thomeé P, et al. A test battery for evaluating hop performance in patients with an ACL injury and patients who have undergone ACL reconstruction. Knee Surg Sports Traumatol Arthrosc 2006;14:778-88.

59 Logerstedt D, Grindem H, Lynch A, et al. Single-legged hop tests as predictors of self-reported knee function after anterior cruciate ligament reconstruction: the Delaware-Oslo ACL cohort study. Am J Sports Med 2012;40:2348-56.

60 Dallinga JM, Benjaminse A, Lemmink KAPM. Which screening tools can predict injury to the lower extremities in team sports?: a systematic review. Sports Med 2012;42:791-815.

61 Herrington L. The effects of 4 weeks of jump training on landing knee valgus and crossover hop performance in female basketball players. $J$ Strength Cond Res 2010;24:3427-32.

62 Barber-Westin SD, Galloway M, Noyes FR, et al. Assessment of lower limb neuromuscular control in prepubescent athletes. Am J Sports Med 2005;33:1853-60.

63 Wright AA, Dischiavi SL, Smoliga JM, et al. Association of lower quarter Y-Balance test with lower extremity injury in NCAA division 1 athletes: an independent validation study. Physiotherapy 2017;103:231-6.

64 Chamari K, Chaouachi A, Hambli M, et al. The five-jump test for distance as a field test to assess lower limb explosive power in soccer players. J Strength Cond Res 2008;22:944-50.

65 Brumitt J, Heiderscheit BC, Manske RC, et al. Preseason functiona test scores are associated with future sports injury in female collegiate athletes. J Strength Cond Res 2018;32:1692-701.

66 Mokha M, Sprague PA, Gatens DR. Predicting musculoskeletal injury in national collegiate athletic association division II athletes from asymmetries and Individual-Test versus composite functional movement screen scores. J Athl Train 2016;51:276-82.

67 Kiesel K, Plisky PJ, Voight ML. Can serious injury in professional football be predicted by a preseason functional movement screen? $N$ Am J Sports Phys Ther 2007;2:147-58.

68 Wright AA, Stern B, Hegedus EJ, et al. Potential limitations of the functional movement screen: a clinical commentary. Br J Sports Med 2016;50:770-1

69 Moran RW, Schneiders AG, Mason J, et al. Do functional movement screen (FMS) composite scores predict subsequent injury? A systematic review with meta-analysis. Br J Sports Med 2017:51:1661-9.

70 Mokkink LB, Terwee CB, Patrick DL, et al. The COSMIN study reached international consensus on taxonomy, terminology, and definitions of measurement properties for health-related patientreported outcomes. J Clin Epidemiol 2010;63:737-45.

71 pp.Prinsen CAC, Mokkink LB, Bouter LM, et al. COSMIN guideline for systematic reviews of patient-reported outcome measures. Quality of Life Research 2018;27:1147-57.

72 Shamseer L, Moher D, Clarke M, et al. Preferred reporting items for systematic review and meta-analysis protocols (PRISMA-P) 2015 elaboration and explanation. BMJ 2015;350:g7647-7.

73 Terwee CB, Jansma EP, Riphagen II, et al. Development of a methodological PubMed search filter for finding studies on measurement properties of measurement instruments. Qual Life Res 2009;18:1115-23.

74 Li T, Higgins JPT, Deeks JJ. Chapter 5: Collecting data. In: Higgins JPT, Thomas J, Chandler J, et al, eds. Cochrane Handbook for systematic reviews of interventions version 6.0 (updated July 2019. Cochrane, 2019. www.training.cochrane.org/handbook

75 Mokkink LB, de Vet HCW, Prinsen CAC, et al. COSMIN risk of bias checklist for systematic reviews of patient-reported outcome measures. Qual Life Res 2018;27:1171-1179.

76 Mokkink LB, Terwee CB, Patrick DL, et al. The COSMIN checklist for assessing the methodological quality of studies on measurement properties of health status measurement instruments: an international Delphi study. Qual Life Res 2010;19:539-49.

77 DerSimonian R, Laird N. Meta-Analysis in clinical trials. Control Clin Trials 1986;7:177-88.

78 Higgins JPT, Thompson SG. Quantifying heterogeneity in a metaanalysis. Stat Med 2002;21:1539-58.

79 Robertson S, Kremer P, Aisbett B, et al. Consensus on measurement properties and feasibility of performance tests for the exercise and sport sciences: a Delphi study. Sports Med Open 2017;3:1-10. 